\title{
Comparison of C-Rich Mira, Semiregular, and Irregular Variables
}

\section{FRANZ KERSCHBAUM ${ }^{1}$, PETER HABISON ${ }^{1,2}$, RITA LOIDL ${ }^{1}$, HANS OLOFSSON ${ }^{3}$, and JOSEF HRON ${ }^{1}$}

\author{
${ }^{1}$ Institut für Astronomie der Universität Wien, Vienna, Austria \\ ${ }^{2}$ Kuffner-Sternwarte, Vienna, Austria \\ ${ }^{3}$ Stockholms Observatorium, Saltsjöbaden, Sweden
}

Carbon-rich Mira variables and semiregular variables of type SRa and SRb as well as irregular variables of type Lb are compared with each other in various aspects. These groups of AGB stars having different pulsational properties are selected from the GCVS and the IRAS Catalogue. Additional near infrared and mm-CO data are used, partly from our own observations. Special emphasis is put on the stars studied by Olofsson et al. (1993, ApJS, 87, 267).

For the four groups of variables, pulsational properties like the amplitude or the period (not for Lbs) are correlated with atmospheric properties like the effective temperature and can be used to separate at least Lbs, SRas and SRbs from the Miras. Whereas the first three groups have relatively high photospheric temperatures $\mathrm{T}^{*}$, medium to low dust temperatures $\mathrm{T}^{\mathrm{d}}$ and relatively high relative sizes of the dust blackbody, the later tend towards lower $\mathrm{T}^{*}$ and somewhat higher $\mathrm{T}^{\mathrm{d}}$. This can be interpreted in the sense that Lbs, SRas and SRbs contain a significant fraction of stars with $60 \mu \mathrm{m}$ excess originating from cold, fossile dust shells and that Miras suffer higher mass loss with perhaps hotter dust temperatures. CO mass-loss rates are well correlated with some results of the BB fits, namely $\mathrm{T}^{*}, \mathrm{~T}^{\mathrm{d}}$ and $\mathrm{L}^{\mathrm{d}} / \mathrm{L}^{*}$. The scale heights and number densities of Lbs would make these stars more massive than the Miras, however this result is based on rather small samples. The DENIS survey will provide much larger volume limited samples of AGB variables and thus allow a check of our results concerning the evolutionary status of Lbs. The main result of that study is that C-rich Lb variables are similar to semiregulars of the same chemistry in many aspects. For the future a more detailed analysis of the individual objects is forseen. This could help to get rid of deviating objects which influence the conclusions from our relatively small samples.

This work is supported by the Fonds zur Förderung der wissenschaftlichen Forschung under project number S7308-AST. 